\title{
Usefulness of delta neutrophil index for early prediction of overt disseminated intravascular coagulopathy in patients with venomous snakebite
}

\author{
Yong Sung Cha', Kang Hyun Lee', Seok Jeong Lee ${ }^{2}$, Ho Chul Kwon', \\ Jong Wook Lee ${ }^{3}$, Hyung II Kim ${ }^{1}$, Oh Hyun Kim ${ }^{1}$, Kyoung-Chul Cha', \\ Hyun Kim ${ }^{1}$, Sung Oh Hwang ${ }^{1}$
}

\begin{abstract}
'Department of Emergency Medicine, Yonsei University Wonju College of Medicine, Wonju, Korea ${ }^{2}$ Department of Internal Medicine, Yonsei University Wonju College of Medicine, Wonju, Korea ${ }^{3}$ Department of Laboratory Medicine, Jincheon Sungmo Hospital, Jincheon, Korea
\end{abstract}

Objective Clinically, consumptive coagulopathy, such as disseminated intravascular coagulopathy (DIC), is the most important among the common venomous snakebite complications owing to the serious hemorrhage risk associated with this condition. We evaluated the predictive value of the delta neutrophil index (DNI)-a new indicator for immature granulocytes-for DIC diagnosis.

Methods This retrospective observational study consecutively assessed adult patients with venomous snakebites for over 51 months. Patients were categorized into the no DIC and DIC groups. DNI values were measured within 24 hours after snakebite.

Results Thirty patients (26.3\%) developed DIC. The DIC group had significantly higher median initial DNI than the no DIC group ( $0 \%$ vs. $0.2 \%, \mathrm{P}<0.001)$. When the DIC group was divided into early and late groups (within and over 24 hours after snakebite, respectively), the DNI of the former was significantly higher than that of the latter and no DIC group. The late DIC group had significantly higher DNI than the no DIC group. Furthermore, DNI positively correlated with the DIC score $(r=0.548, P<0.001)$. The initial DNI (odds ratio, 4.449; 95\% confidence interval, 1.738 to 11.388; $P=0.002$ ) was an early DIC predictor. The area under the curve based on the initial DNI's receiver operating characteristic curve was 0.724 .

Conclusion DNI values were significantly higher in the DIC group. Additionally, DNI was an early predictor of DIC development in patients with venomous snakebites in the emergency department.

Keywords Snake bites; Disseminated intravascular coagulopathy; Diagnosis; Biomarker
Received: 24 November 2016

Revised: 1 February 2017

Accepted: 7 February 2017

Correspondence to: Kang Hyun Lee Department of Emergency Medicine, Yonsei University Wonju College of Medicine, 20 Ilsan-ro, Wonju 26426, Korea

E-mail: ed119@yonsei.ac.kr

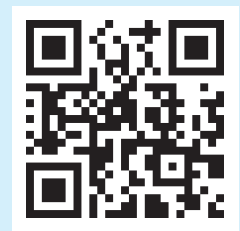

How to cite this article:

Cha YS, Lee KH, Lee SJ, Kwon HC, Lee JW, Kim HI, Kim OH, Cha KC, Kim H, Hwang SO. Usefulness of delta neutrophil index for early prediction of overt disseminated intravascular coagulopathy in patients with venomous snakebite. Clin Exp Emerg Med 2018;5(2):76-83.
This is an Open Access article distributed under the terms of the Creative Commons Attribution Non-Commercial License (http:// creativecommons.org/licenses/by-nc/4.0/). 


Capsule
Wumat is already known
Treatment strategies for venomous snakebites generally focus on resolving coagulation abnormalities. Clinically, con-
sumptive coagulopathy, including disseminated intravascular coagulopathy (DIC), is the most important among the
snakebite complications because it can result in severe and life-threatening hemorrhage.
$\begin{aligned} & \text { What is new in the current study } \\ & \text { Delta neutrophil index values were significantly higher in the DIC group. Furthermore, delta neutrophil index was an } \\ & \text { early predictor for DIC development in patients with venomous snakebites in the emergency department. }\end{aligned}$

\section{INTRODUCTION}

Approximately 421,000 venomous snakebites occur worldwide each year, resulting in 20,000 deaths. Four types of venomous snakes have been identified in Korea: Gloydius brevicaudus, Gloydius ussuriensis, and Gloydius intermedius from the family Viperidae and Rhabdophis tigrinus from the family Colubridae.' In Korea, approximately 192 to 621 venomous snakebites are reported annually, which account for five deaths. ${ }^{2,3}$

The toxic manifestations of venomous snakebites include local tissue damage, such as soft tissue edema, necrosis, or chemically mediated inflammation, and major systemic complications, such as neurotoxicity, myotoxicity, and cytotoxicity. The treatment strategies for venomous snakebites generally focus on resolving coagulation abnormalities. ${ }^{4}$ Clinically, consumptive coagulopathy, including disseminated intravascular coagulopathy (DIC), is the most important among the snakebite complications because it can result in severe and life-threatening hemorrhage. ${ }^{5}$

Some reports have shown that the delta neutrophil index (DNI)-which is a new indicator for immature granulocytes and a commonly evaluated consumptive coagulopathy marker in sepsis-is associated with DIC in critically ill patients with suspected sepsis. ${ }^{6-8}$ The DNI is defined as the difference in leukocyte subfractions identified via a cytochemical myeloperoxidase reaction and nuclear lobularity assay and is measured using a blood cell analyzer (ADVIA 120; Siemens Healthcare Diagnostics, Tarrytown, NY, USA). It is strongly correlated with manual immature granulocyte counts, which are difficult to obtain in clinical practice because of the inaccuracy of manual counting. ${ }^{6,79-13}$ One advantage offered by the DNI is that the tests are simultaneously performed with routine complete blood count testing without additional cost.

We hypothesized that the initial DNI (measured within 24 hours after snakebite) might be higher in patients with DIC manifesting as consumptive coagulopathy in the case of a venomous snakebite. Therefore, this study aimed to investigate the differences in initial DNI in relation to the presence of DIC. Moreover, the usefulness of initial DNI as an early DIC predictor in patients with venomous snakebites was examined.

\section{METHODS}

\section{Study subjects and setting}

This retrospective observational study included adults aged more than 18 years with venomous snakebite who were consecutively treated for over a 51-month period (from 2011 May to 2015 July) from the time when the DNI checks were implemented. In this study, the emergency department (ED) was located in a single urban, tertiary-care hospital, which has an annual visit volume of over 45,000 and is staffed 24 hours per day by board-certified emergency physicians.

All patients with the word "snake," "venomous," or "envenoming/envenomation" in the ED discharge codes registered in computerized hospital records were initially considered. The diagnostic criteria for venomous snakebite included one or more of the following: 1) a confirmed two-fang wound, 2) a bite from a snake with a triangular-shaped head, 3) venomous snakebite confirmation based on the patient's experience, and 4) presence of abnormal physical and laboratory findings, such as shock, tachypnea, bleeding, thrombocytopenia, DIC, or diplopia, despite other insufficient findings. ${ }^{14}$ Antivenin was administered when the patients had systemic signs, including collapse, convulsions, weakness, paralysis, respiratory failure, shock, or bleeding, or severe local symptoms associated with snakebite. The dose was repeated if the symptoms persisted.

The exclusion criteria were as follows: 1) use of granulocyte colony-stimulating factors, glucocorticoids, or other immunosuppressants that could affect the DNI values; 2 ) presence of hematological abnormalities and evidence of wound infection, such as redness, localized heat sensation, or purulent discharge, and ma- 
jor trauma that may influence the DNI values; 3) use of anticoagulants and presence of liver cirrhosis, which could affect the coagulation profiles, such as prothrombin time (PT); 4) presentation more than 24 hours after snakebite; 5) transfer of patient from other hospitals after antivenin use, which may exert effects on the DNI that could not be excluded; and 6) transfer of patient to another hospital regardless of ED admission or not.

Patients were categorized as having DIC or none. The DIC group included patients with consumptive coagulopathy through activation of the entire clotting pathway, such as DIC. We used a diagnostic algorithm for DIC that was proposed by the International Society on Thrombosis and Haemostasis. The algorithm parameters include the platelet count $(>100,000 / \mu \mathrm{L}=0,<100,000 /$ $\mu \mathrm{L}=1,<50,000 / \mu \mathrm{L}=2$ ); fibrin degradation product, such as $d-$ dimer (no increase $=0$, moderate increase $=2$, strong increase $=3$ ); prolonged $\mathrm{PT}(<3 \mathrm{sec}=0,>3 \mathrm{sec}=1,>6 \mathrm{sec}=2)$; and fibrinogen level $(>1.0 \mathrm{~g} / \mathrm{dL}=0,<1.0 \mathrm{~g} / \mathrm{dL}=1)$. A score of 5 or higher is consistent with the presence of $\mathrm{DIC}$, and this algorithm has been reported to have a sensitivity and specificity of 91\% and 97\%, respectively, for DIC diagnosis. ${ }^{15,16}$ We reclassified patients with DIC into early DIC (DIC within 24 hours after snakebite) and late DIC (DIC beyond 24 hours after snakebite) groups.

\section{Data collection}

The data were collected through a retrospective review of the patients' electronic medical records by two emergency physicians who were blinded to the study objectives and hypothesis. The abstractors were blinded to the patient group categorization, which was accomplished by another emergency physician. Additionally, the abstractors were trained prior to data collection to reduce the possible bias from the data collection procedure. We used explicit case report forms. The chart abstractors and study coordinators periodically met to resolve any disputes and review coding rules. The study coordinators monitored the abstractors' performance. Considering that the study was performed retrospectively and observationally, the patients' records and information were anonymously processed prior to analysis. The following information was obtained from the medical records: age, sex, site of the bite, time from snakebite to ED arrival, underlying diseases, initial systolic blood pressure, severity as determined by a traditional snakebite severity grading scale (Table 1), ${ }_{1}^{17}$ antivenin use, and initial symptoms and signs, including both local and systemic manifestations. Systemic complications, including hematological (anemia, ${ }^{18}$ thrombocytopenia, PT/partial thrombin time [PTT] prolongation, and bleeding), neurological (blurred vision, diplopia, and dizziness), and other complications (rhabdomyolysis, acute kidney injury $[\mathrm{AKI}]_{1}^{19,20}$ and shock [systolic blood pressure $<90$ $\mathrm{mmHg}]$ ), occurring during hospitalization were investigated.

Blood samples for the analyses of serum white blood cell (WBC) counts, C-reactive protein levels, DNI, platelet counts, and fibrinogen, PT, PTT, d-dimer, and antithrombin III levels were obtained through venipuncture within the first hour of ED admission. An automatic cell analyzer (ADVIA 120/2120, Siemens Healthcare Diagnostics) was used to measure the DNI. The technique is specific to the technology found in the ADVIA unit manufactured by Siemens Healthcare Diagnostics. This flow cytometry-based hematological analyzer utilizes two independent WBC analysis methods by using myeloperoxidase and lobularity/nuclear density channels. The DNI was calculated in leukocyte differentials using the following formula: DNI = (leukocyte subfraction assayed in the myeloperoxidase channel via cytochemical reaction) - (leukocyte subfraction counted in the nuclear lobularity channel via reflected light beam). ${ }^{7,12,13}$

The primary outcome of this study was the difference in the initial DNI measured in the ED in relation to the presence of DIC in patients with venomous snakebites. The secondary outcome was the predictive ability of the initial DNI on the presence of DIC upon presentation at the ED. This study was approved by the Institutional Review Board of our medical college (CR316051). The informed consent was waived due to retrospective design.

\section{Statistical analysis}

Categorical variables are presented as frequencies and percentages, whereas continuous variables are presented as means and standard deviations or medians and interquartile ranges. Chisquare or Fisher's exact tests were used to compare categorical

Table 1. Traditional grading scale for snakebite severity

\begin{tabular}{ll}
\hline Severity scale & \multicolumn{1}{c}{ Manifestations } \\
\hline 0 (no envenomation) & Absence of local or systemic signs or symptoms \\
I (minimal) & Local swelling, absence of systemic signs, and normal laboratory findings \\
II (moderate) & Swelling extending past site of bite (6-12 inches), $\geq 1$ systemic signs or symptoms, and abnormal laboratory findings \\
III (severe) & Marked swelling (>12 inches), tissue loss, multiple or severe systemic symptoms, immediate systemic signs, and rapid progression of symptoms \\
IV (very severe) & $\begin{array}{l}\text { Rapid development of local reaction, ecchymosis, necrosis, blebs, blisters, swelling severe enough to obstruct venous or arterial flow, and swelling } \\
\text { may involve the ipsilateral trunk }\end{array}$
\end{tabular}


variables, whereas two-sample t-tests or Mann-Whitney U-tests were utilized to compare continuous variables. To compare three groups, we performed the chi-square test (Fisher exact test), analysis of variance, or Kruskal-Wallis test, as appropriate. We also analyzed multiple comparisons using Bonferroni correction, Sheffé's method, and Dunn's procedure, as appropriate. Normality was first assessed using the Shapiro-Wilk test. Correlation coefficients (r) were calculated via the Spearman's method. Logistic regression analysis was used to identify the factors that predicted the presence of DIC. The results were expressed as odds ratio (OR) with 95\% confidence intervals (Cls). The area under the receiver operating characteristic curve was utilized to evaluate the DNI's predictive ability in classifying DIC. P-values less than 0.05 were considered statistically significant, and all statistical analyses were conducted using IBM SPSS Statistics ver. 23.0 (IBM Corp., Armonk, NY, USA).

\section{RESULTS}

\section{Patient characteristics}

A total of 170 patients with venomous snakebites were consecutively assessed during the study period. Among these patients, 56 were excluded based on the following criteria: ED arrival with wound infection (one patient), presentation beyond 24 hours following bite (20 patients), transferred from other hospital after antivenin use (20 patients), transferred to other hospital regardless of ED admission or not (six patients), discharged against medical advice (two patients), and insufficient data (seven patients). After exclusion, we included a total of 114 patients with venomous snakebites.

Of the 114 analyzed patients, $76(66.7 \%)$ were men with a median age of 56 years (range, 18 to 93 years). The median time from snakebite to ED arrival was 3 hours. Hypertension (28 patients, 25.7\%) was the most common underlying disease. Patients with bite severity grade of II (56 patients, 50.5\%) were most com-

Table 2. Baseline characteristics and laboratory findings of patients with venomous snakebite

\begin{tabular}{|c|c|c|c|}
\hline Variable & No DIC group $(n=84)$ & DIC group $(n=30)$ & P-value \\
\hline Age (yr) & $56(42-67)$ & $58(55-63)$ & 0.342 \\
\hline Sex, male & $48(57.1)$ & 28 (93.3) & $<0.001$ \\
\hline Site of bite & & & 0.271 \\
\hline Hand & $54(64.3)$ & 25 (83.3) & \\
\hline Leg & $6(7.1)$ & $1(3.3)$ & \\
\hline Foot & $18(21.4)$ & 4 (13.3) & \\
\hline Time from snakebite to ED arrival ( $\mathrm{hr}$ ) & $2.0(1.0-6.8)$ & $5.0(2.3-18)$ & 0.002 \\
\hline \multicolumn{4}{|l|}{ Underlying disease } \\
\hline Diabetes mellitus & 7 (8.9) & $3(10.0)$ & 1.000 \\
\hline Hypertension & $20(25.3)$ & $8(26.7)$ & 0.885 \\
\hline Cardiac disease & $4(4.8)$ & $1(3.3)$ & 1.000 \\
\hline Systolic blood pressure & $146(127-159)$ & $137(114-161)$ & 0.294 \\
\hline Final severity & & & $<0.001$ \\
\hline I & $36(44.4)$ & $0(0)$ & \\
\hline ॥ & $36(44.4)$ & $20(66.7)$ & \\
\hline III & $0(0)$ & $6(20.0)$ & \\
\hline IV & $9(11.1)$ & $4(13.3)$ & \\
\hline Antivenin & & & $<0.001$ \\
\hline None & $28(33.3)$ & $1(11.1)$ & \\
\hline Single & 48 (57.1) & $5(55.5)$ & \\
\hline Multidose & 8 (9.5) & $3(33.3)$ & \\
\hline \multicolumn{4}{|l|}{ Local symptoms and signs } \\
\hline Pain & 78 (98.7) & $29(100)$ & 1.000 \\
\hline Blisters & $9(17.3)$ & 4 (20.0) & 0.746 \\
\hline Severe swelling & 64 (85.3) & 26 (89.7) & 0.752 \\
\hline Discoloration & $30(50.8)$ & $15(65.2)$ & 0.240 \\
\hline \multicolumn{4}{|l|}{ Inflammatory markers } \\
\hline White blood cell count & $7,635(6,113-9,800)$ & $9,800(6,615-15,145)$ & 0.017 \\
\hline C-reactive protein & $0.29(0.29-0.30)$ & $0.29(0.29-0.51)$ & 0.630 \\
\hline Delta neutrophil index & $0(0-0)$ & $0.2(0-2.2)$ & $<0.001$ \\
\hline
\end{tabular}

Values are presented as numbers (range) or numbers (\%).

DIC, disseminated intravascular coagulopathy; ED, emergency department. 
mon, and 81 patients (71.1\%) were treated using intravenous antivenin. The common local symptoms at initial presentation included pain (107 patients, 99.1\%) and edema (90 patients, 86.5\%), whereas the common systemic symptom was nausea (seven patients, 6.3\%). Among the hematological complications, anemia (42 patients, 36.8\%) was the most common.

\section{Comparisons of general characteristics and laboratory findings based on the presence of DIC}

Thirty patients (26.3\%) developed DIC. Of these patients, nine developed early DIC (DIC within 24 hours after snakebite), and 21

Table 3. Systemic symptoms, signs, and complications

\begin{tabular}{lccr}
\hline Variable & $\begin{array}{c}\text { No DIC group } \\
(\mathrm{n}=84)\end{array}$ & $\begin{array}{c}\text { DIC group } \\
(\mathrm{n}=30)\end{array}$ & P-value \\
\hline $\begin{array}{l}\text { Systemic symptoms and signs } \\
\text { Nausea }\end{array}$ & $3(3.8)$ & $4(13.3)$ & 0.089 \\
Headache & $2(2.5)$ & $2(6.7)$ & 0.303 \\
Decreased mental status & $0(0)$ & $2(6.7)$ & 0.075 \\
$\quad$ Dyspnea & $4(5.1)$ & $1(6.7)$ & 0.666 \\
Hematological complications & & & \\
Anemia & $26(31.0)$ & $16(53.3)$ & 0.029 \\
Thrombocytopenia & $13(15.5)$ & $16(53.3)$ & $<0.001$ \\
PT/PTT prolongation & $8(9.5)$ & $16(53.3)$ & $<0.001$ \\
$\quad$ Bleeding & $1(1.2)$ & $5(16.7)$ & 0.005 \\
Neurological complications & & & \\
$\quad$ Blurred vision/diplopia & $3(3.8)$ & $0(0)$ & 0.560 \\
$\quad$ Dizziness & $8(10.1)$ & $3(10.0)$ & 1.000 \\
Other complications & & & \\
$\quad$ Rhabdomyolysis & $10(12.7)$ & $9(30.0)$ & 0.033 \\
Acute kidney injury & $0(0)$ & $3(10.0)$ & 0.019 \\
Shock & $1(1.3)$ & $2(6.7)$ & 0.180 \\
\hline
\end{tabular}

Values are presented as numbers (\%).

DIC, disseminated intravascular coagulopathy; PT, prothrombin time; PTT, partial thrombin time. developed late DIC (DIC beyond 24 hours after snakebite). Tables 2 and 3 show the comparisons of baseline characteristics and inflammatory markers. Patients in the no DIC and DIC groups differed significantly in the proportion of males (57.1\% vs. 93.3\%, $\mathrm{P}<0.001)$. The DIC group had significantly longer time from snakebite to ED arrival than the no DIC group (5.0 vs. 2.0 hours, $P=0.002$ ). Furthermore, the DIC group had significantly higher final bite severity $(P<0.001)$ and more frequent multidose antivenin administrations $(P<0.001)$ than the no DIC group. All hematological complications, including anemia, thrombocytopenia, PT/PTT prolongation, and bleeding, were significantly more common in the DIC group than in the no DIC group. However, no significant differences in neurological complications were observed between the two groups. Patients with DIC had significantly higher rhab-

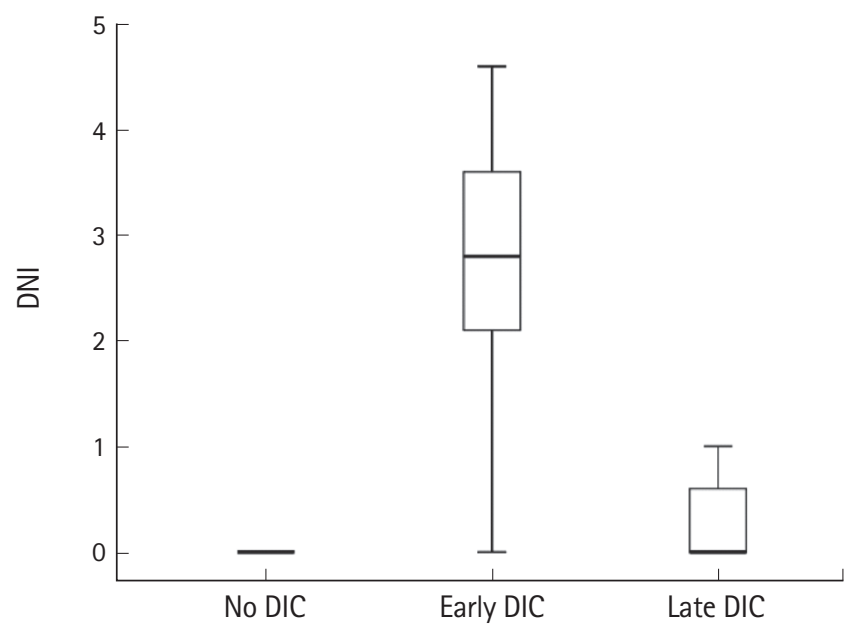

Fig. 1. Median value of delta neutrophil index (DNI) in relation to the presence and onset time of disseminated intravascular coagulopathy (DIC).

Table 4. Median values of inflammatory markers based on the presence and onset time of DIC

\begin{tabular}{|c|c|c|c|c|}
\hline Inflammatory marker & No DIC group $(n=84)$ & Early DIC group $(n=9)$ & Late DIC group $(n=21)$ & P-value \\
\hline DIC score & $1(1-1.75)^{a, b)}$ & $5(5-6)^{a)}$ & $5(5-6)^{b)}$ & $<0.001$ \\
\hline Time to development of DIC ( $\mathrm{hr}$ ) & & & & $<0.001$ \\
\hline$<24$ & 0 & $9(100 \%)$ & 0 & \\
\hline $24-72$ & 0 & 0 & $18(85.7 \%)$ & \\
\hline$>72$ & 0 & 0 & $3(14.3 \%)$ & \\
\hline Platelet $(/ \mu \mathrm{L})$ & $254,000(207,000-299,750)^{\mathrm{a}, \mathrm{b})}$ & $90,000(67,500-121,000)^{a)}$ & $95,000(90,000-258,500)^{b)}$ & $<0.001$ \\
\hline PT (sec) & $1(0.9-1)^{a, b)}$ & $6(3.3-9.5)^{\mathrm{a})}$ & $8(1-10)^{\mathrm{b})}$ & $<0.001$ \\
\hline D-dimer (ng/mL) & $156(74-312)^{a, b)}$ & $3,459(2,703-7,413)^{a, c}$ & $1,220(780-2,145)^{b, c)}$ & $<0.001$ \\
\hline Fibrinogen (g/dL) & $357(301-439)^{a, b)}$ & $154(60-225)^{a)}$ & $202(60-332)^{b)}$ & $<0.001$ \\
\hline WBC count & $7,635(6,113-9,800)^{a)}$ & $16,030(7,910-19,120)^{\mathrm{a}}$ & $9,480(6,345-13,980)$ & 0.015 \\
\hline C-reactive protein & $0.29(0.29-0.30)$ & $0.29(0.29-0.46)$ & $0.29(0.29-0.86)$ & 0.877 \\
\hline DNI & $0(0-0)^{a, b)}$ & $2.8(1.7-4.0)^{\mathrm{a}, \mathrm{cc}}$ & $\left.0(0-0.8)^{b, c}\right)$ & $<0.001$ \\
\hline
\end{tabular}

DIC, disseminated intravascular coagulopathy; PT, prothrombin time; WBC, white blood cell; DNI, delta neutrophil index.

${ }^{a)}$ No DIC group vs. early DIC group (DIC score, $\mathrm{P}<0.001$; platelet, $\mathrm{P}<0.001$; $\mathrm{PT}, \mathrm{P}<0.001$; d-dimer, $\mathrm{P}<0.001$; fibrinogen, $\mathrm{P}<0.001 ; \mathrm{WBC}, \mathrm{P}=0.008$; $\mathrm{DNI}, \mathrm{P}<0.001$ ). ${ }^{\text {b) No }} \mathrm{DIC}$

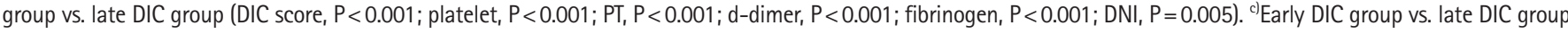
(d-dimer, $\mathrm{P}=0.003 ; \mathrm{DNI}, \mathrm{P}<0.001)$. 
Table 5. Predictors for the presence of overt disseminated intravascular coagulopathy as determined by multivariate logistic regression analysis

\begin{tabular}{lcc}
\hline Variable & \multicolumn{1}{c}{ OR $(95 \% \mathrm{Cl})$} & P-value \\
\hline Male & $5.785(1.155-28.964)$ & 0.033 \\
Time from snakebite to ED arrival (hr) & $1.110(1.036-1.189)$ & 0.003 \\
White blood cell count & $1.000(1.000-1.000)$ & 0.695 \\
Delta neutrophil index & $4.449(1.738-11.388)$ & 0.002 \\
\hline
\end{tabular}

$\mathrm{OR}$, odds ratio; $\mathrm{Cl}$, confidence interval; $\mathrm{ED}$, emergency department.

domyolysis and AKI incidences than those with no DIC $(P=0.033$ and 0.019 , respectively).

The DIC group had significantly higher median initial serum WBC and DNI values than the no DIC group $(7,635$ vs. 9,800, $P=0.017 ; 0$ vs. $0.2, P<0.001$, respectively). The initial serum $C-$ reactive protein levels did not differ significantly between groups. When the DIC group was divided into the early and late groups, the mean DNI value of the former was significantly higher than that of the latter and no DIC group. The late DIC group had significantly higher DNI values than the no DIC group. Additionally, the early DIC group has significantly higher WBC counts than the no DIC group (Table 4 and Fig. 1). DNI was positively correlated with DIC score $(r=0.548, P<0.001)$.

Multiple logistic regression analyses were conducted to identify the early predictors related to DIC development, such as male sex, time from snakebite to ED arrival, serum WBC, and DNI. Male sex $(0 R, 5.785 ; 95 \% \mathrm{Cl}, 1.155$ to $28.964 ; \mathrm{P}=0.033)$, time from snakebite to $E D$ arrival $(O R, 1.110 ; 95 \% \mathrm{Cl}, 1.036$ to $1.189 ; \mathrm{P}=$ 0.003), and initial DNI $(\mathrm{OR}, 4.449 ; 95 \% \mathrm{Cl}, 1.738$ to $11.388 ; \mathrm{P}=$ 0.002) were found to be statistically associated with DIC development. The area under the curve based on the initial DNI's receiver operating characteristic curve was 0.724 (Table 5 and Fig. 2).

\section{DISCUSSION}

In this study, the initial DNI (measured within 24 hours after snakebite) was significantly higher in the DIC group, and the DNI was positively correlated with the DIC score. Additionally, the initial DNI was a significant early predictor of DIC development. Our findings may be explained by the following: 1) during stress, inflammation, or infection, immature granulocytes enter the circulation. ${ }^{10,21}$ Therefore, venomous snakebites can cause increased $\mathrm{DNI}$, which is strongly correlated with increased manual immature granulocyte counts. This finding can be attributed to the inflammatory reaction caused by envenomation, which is the major characteristic feature of bites from viperine species. ${ }^{22,23}$ 2) Both the venomous toxin itself and the inflammatory reaction caused by viperine envenomation can cause DIC. The inflammatory reac-

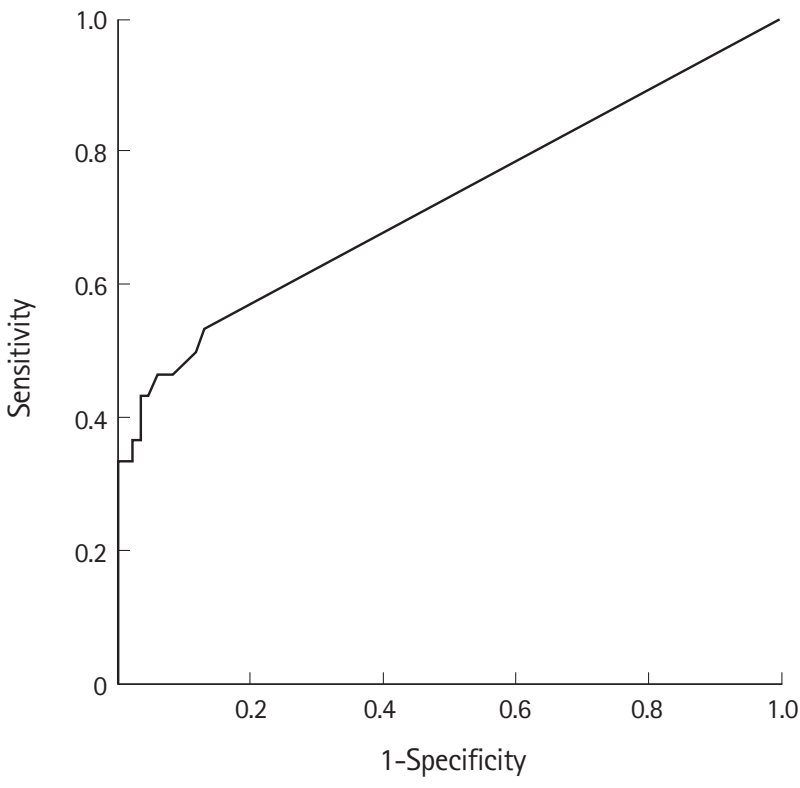

Fig. 2. Receiver operating characteristic curve of delta neutrophil index for disseminated intravascular coagulopathy prediction.

tion may contribute to more severe DIC development than the toxin itself. 3) DNI is associated with hypercoagulable states, such as DIC. Based on the reports on sepsis, DNI was closely related to the presence of DIC, which is one of the sepsis complications. Furthermore, Nahm et al. ${ }^{7}$ and Park et al. ${ }^{8}$ suggested that DNI might be linked to a hypercoagulable state associated with sepsis. We thought that the hypercoagulable state caused by venomous snakebites might be related to DNI elevation, similar to sepsis. Therefore, increased DNI may not only develop due to venomous snakebites but may also be significantly higher in the DIC group. Thus, DNI can be correlated with DIC score.

However, some venomous toxins, such as thrombin-like enzymes (TLEs) or fibrinogenases, can result in consumptive coagulopathy with low fibrinogen levels but normal levels of other clotting factors, unlike DIC. This finding may be attributed to the cleaving property of either the $\alpha$ - or $\beta$-chains of fibrinogen that transforms into fibrinopeptide $A$ or $B$, resulting in fibrinogen consumption without fibrin formation. ${ }^{24,25}$ Therefore, we could not assess the occurrence of consumptive coagulopathy by measuring the TLE or fibrinogenase levels using the DIC algorithm, although no information is available on the ratio of consumptive coagulopathy caused by TLEs or fibrinogenases among all cases of consumptive coagulopathy resulting from venomous snakebites in Korea.

In this study, the hematological complication prevalence (58 patients, 50.9\%) was similar to that reported in previous studies (7\% to $82.4 \%){ }^{26-28}$ The time from snakebite to ED arrival was 
significantly higher in the DIC group and was related to DIC development. This difference may be caused by the delay in management, such as antivenin administration, after venomous snakebites that could allow the condition to progress. Therefore, DIC should be suspected in patients arriving at the ED later than a few hours after venomous snakebites. The DIC group had higher bite severity and antivenin use than the no DIC group. This finding suggests that patients with systemic reactions, for example, hematological complications, including DIC, naturally have a high bite severity. Similarly, antivenin is used more often in patients with systemic reactions, such as hematological complications. Sex-specific differences between the two groups could not be explained. In this study, men and women did not differ significantly in terms of age, time from snakebite to ED arrival, and bite severity (data not shown). However, men had longer median time from snakebite to ED arrival than women did, although the difference was not significant ( 3.0 vs. 1.8 hours, $P=0.101$ ). These variations may contribute to differences in the DIC development. Serum WBC count was not a predictor of DIC development, although it significantly differed between the no DIC and DIC groups.

This study had several limitations. First, it was constrained by its retrospective design and missing data, including the type of venomous snakes, during collection. Second, the sample size was possibly small because this study was conducted at the emergency center of a single hospital. However, we investigated all patients with venomous snakebites from the time when the DNI levels were measured, starting in May 2011, to reduce this possible bias. Third, we could not assess the presence of consumptive coagulopathy by measuring the TLE or fibrinogenase levels based on the DIC algorithm..$^{25}$ Finally, we could not determine the direct relationship between DNI and consumptive coagulopathy because of the influence of the toxin itself on the DNI values. A well-designed prospective study is necessary to overcome these limitations.

In summary, DNI values were significantly higher in the DIC group. Moreover, DNI served as an early predictor of DIC development in patients with venomous snakebites in the ED.

\section{CONFLICT OF INTEREST}

No potential conflict of interest relevant to this article was reported.

\section{REFERENCES}

1. Shim JH, Son YJ, Lee SS, Park KS, Oh HB, Park YD. Ecological study on poisonous snake and investigation of the venom characteristics, snakebiting frequency in Korea. Korean J Environ Ecol 1998;12:58-77.

2. Chippaux JP. Snake-bites: appraisal of the global situation. Bull World Health Organ 1998;76:515-24.

3. Kasturiratne A, Wickremasinghe AR, de Silva N, et al. The global burden of snakebite: a literature analysis and modelling based on regional estimates of envenoming and deaths. PLoS Med 2008;5:e218.

4. Agarwal R, Singh AP, Aggarwal AN. Pulmonary oedema complicating snake bite due to Bungarus caeruleus. Singapore Med J 2007;48:e227-30.

5. Isbister GK. Snakebite doesn't cause disseminated intravascular coagulation: coagulopathy and thrombotic microangiopathy in snake envenoming. Semin Thromb Hemost 2010;36: 444-51.

6. Seok Y, Choi JR, Kim J, et al. Delta neutrophil index: a promising diagnostic and prognostic marker for sepsis. Shock 2012; 37:242-6.

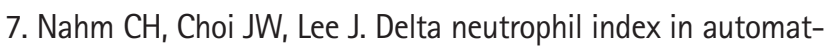
ed immature granulocyte counts for assessing disease severity of patients with sepsis. Ann Clin Lab Sci 2008;38:241-6.

8. Park BH, Kang YA, Park MS, et al. Delta neutrophil index as an early marker of disease severity in critically ill patients with sepsis. BMC Infect Dis 2011;11:299.

9. Ansari-Lari MA, Kickler TS, Borowitz MJ. Immature granulocyte measurement using the Sysmex XE-2100. Relationship to infection and sepsis. Am J Clin Pathol 2003;120:795-9.

10. Cornbleet PJ. Clinical utility of the band count. Clin Lab Med 2002;22:101-36.

11. Kim HW, Ku S, Jeong SJ, et al. Delta neutrophil index: could it predict mortality in patients with bacteraemia? Scand J Infect Dis 2012;44:475-80.

12. Kratz A, Maloum K, O'Malley C, et al. Enumeration of nucleated red blood cells with the ADVIA 2120 Hematology System: an International Multicenter Clinical Trial. Lab Hematol 2006;12:63-70.

13. Harris N, Jou JM, Devoto G, et al. Performance evaluation of the ADVIA 2120 hematology analyzer: an international multicenter clinical trial. Lab Hematol 2005;11:62-70.

14. Park EJ, Yoon SK, Ahn JH, et al. Systemic complications occurring after Korean venomous snake bite, with focus on hematologic and neurologic complications. J Korean Soc Clin Toxicol 2009;7:90-6.

15. Levi $\mathrm{M}$, Toh $\mathrm{CH}$, Thachil J, Watson HG. Guidelines for the diagnosis and management of disseminated intravascular coagulation. British Committee for Standards in Haematology. Br J Haematol 2009;145:24-33. 


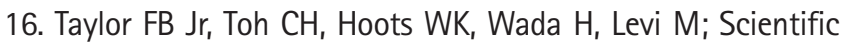
Subcommittee on Disseminated Intravascular Coagulation (DIC) of the International Society on Thrombosis and Haemostasis (ISTH). Towards definition, clinical and laboratory criteria, and a scoring system for disseminated intravascular coagulation. Thromb Haemost 2001;86:1327-30.

17. Dart RC, Hurlbut KM, Garcia R, Boren J. Validation of a severity score for the assessment of crotalid snakebite. Ann Emerg Med 1996;27:321-6.

18. World Health Organization. Worldwide prevalence of anaemia 1993-2005: WHO global database on anaemia [Internet]. Geneva: World Health Organization; 2008 [cited 2017 Apr 11]. Available from: http://apps.who.int/iris/bitstream/10665/ 43894/1/9789241596657_eng.pdf.

19. Melli G, Chaudhry V, Cornblath DR. Rhabdomyolysis: an evaluation of 475 hospitalized patients. Medicine (Baltimore) 2005;84:377-85.

20. Mehta RL, Kellum JA, Shah SV, et al. Acute Kidney Injury Network: report of an initiative to improve outcomes in acute kidney injury. Crit Care 2007;11:R31.

21. Smith JA. Neutrophils, host defense, and inflammation: a double-edged sword. J Leukoc Biol 1994;56:672-86.
22. Rosenfeld G. Symptomatology, pathology and treatment of snake bites in South America. In: Bucherl W, Buckley EE, editors. Venomous animals and their venoms. New York, NY: Academic Press; 1971. p.345-84.

23. Sawai Y. Studies on snakebites in the Asian areas. In: Eaker D, Wadstrom T, editors. Natural toxins: proceedings of the 6th International Symposium on Animal, Plant, and Microbial Toxins; 1979 Aug. Uppsala: Pergamon; 1980. p.25-32.

24. Lu Q, Clemetson JM, Clemetson KJ. Snake venoms and hemostasis. J Thromb Haemost 2005;3:1791-9.

25. Isbister GK. Procoagulant snake toxins: laboratory studies, diagnosis, and understanding snakebite coagulopathy. Semin Thromb Hemost 2009;35:93-103.

26. Lee BJ, Hong SI, Kim HS, et al. Hematological features of coagulopathy and the efficacy of antivenin therapy for a Korean snakebite. J Korean Surg Soc 2007;72:18-26.

27. Isbister GK, Williams V, Brown SG, White J, Currie BJ; Australian Snakebite Project Investigators. Clinically applicable laboratory end-points for treating snakebite coagulopathy. Pathology 2006;38:568-72.

28. White J. Snake venoms and coagulopathy. Toxicon 2005;45: 951-67. 